\title{
IMPLICAÇÕES NUTRICIONAIS DA DIETA VEGETARIANA DA INTRODUÇÃO ALIMENTAR À IDADE PRÉ-ESCOLAR
}

\author{
NUTRITIONAL IMPLICATIONS OF THE VEGETARIAN DIET OF FOOD \\ INTRODUCTION TO PRESCHOOL AGE
}

\author{
Ana Karolyny Ferreira da Silva ${ }^{1}$ \\ Bárbara Granato Russo ${ }^{2}$ \\ Dryelle Oliveira Dias Leão ${ }^{3}$ \\ Laiane Neves da Silva ${ }^{4}$ \\ Taíse Cristina Matos Marques ${ }^{5}$
}

RESUMO: OBJETIVO: Reunir evidências científicas acerca dos benefícios e malefícios do vegetarianismo na introdução alimentar de crianças até a idade préescolar. MÉTODO: Foram utilizadas quatro bases de dados para investigação: Literatura Latino Americana e do Caribe em Ciências da Saúde (Lilacs), Scientific Electronic Library Online (SciELO), National Library of Medicine (PubMED) e Google Acadêmico, utilizando como descritores dieta vegetariana, desenvolvimento cognitivo, crescimento, crianças, alimentação complementar, deficiências nutricionais, riscos e benefícios, restritos ao período de 2011 até 2021. A busca resultou em 69 artigos e depois de aplicados os critérios de inclusão e exclusão foram selecionados 11. RESULTADOS: Observou-se que a dieta vegetariana pode ser adotada desde a fase da alimentação complementar pois pode contribuir para a redução no risco de obesidade, menor incidência de casos de hipertensão e doenças coronarianas, alguns tipos decâncer e ainda o diabetes com início na fase adulta, além de promover adequado crescimento e desenvolvimento cognitivo da criança,ao ser realizado com auxílio de profissionais que possam adequar micronutrientes para reduzir os riscos de deficiências nutricionais principalmente em relação a vitamina B12 e ferro. CONCLUSÃO: É possível incluir a dieta vegetariana no hábito alimentar de crianças desde que seja realizado o acompanhamento de um profissional de saúde habilitado que possa adequar todos os macro e

\footnotetext{
${ }^{1}$ Nutricionista, graduada pela instituição UNINASSAU, Teresina-PI; Pós-Graduanda pela instituição GANEP Educação, São Paulo-SP.

${ }^{2}$ Graduação em Nutrição pela Universidade Cruzeiro do Sul, São Paulo, São Paulo, Brasil.

${ }^{3}$ Nutricionista, mestranda do Programa de Pós-Graduação em Nutrição Humana, Faculdade de Ciências da Saúde, Universidade de Brasília. Brasília-DF.

${ }^{4}$ Nutricionista, Graduação em Nutrição pela Universidade Cruzeiro do Sul, São Paulo, SãoPaulo, Brasil.

${ }^{5}$ Acadêmica de Nutrição na FIMCA - Centro Universitário Aparício Carvalho. Porto Velho-RO.
} 
micronutrientes que são fundamentais desde a introdução alimentar atéa idade préescolar.

Palavras chave: Alimentação Complementar. Benefícios. Dieta vegetariana.

ABSTRACT: OBJECTIVE: To gather scientific evidence about the benefits and harms of vegetarianism in introducing food to children up to preschool age. METHOD: Four databases were used for research: the Latin American and Caribbean Literature in Health Sciences (Lilacs), Scientific Electronic Library Online (SciELO), National Library of Medicine (PubMED) and Google Scholar; using descriptors like vegetarian diet, cognitive development, growth, children, complementary feeding, nutritional deficiencies, risks and benefits, limited for 2011 to 2021. The search resulted in 69 articles and after applying the inclusion and exclusion criteria, 11 articles were selected. RESULTS: It was observed that vegetarian diet can be adopted from the stage of complementary feeding because it can contribute to the reduction in the risk of obesity, lower incidence of cases of hypertension and coronary heart disease, some types of cancer and even diabetes with onset in adulthood; It can also promoting adequate growth and cognitive development of the child, when performed with the help of professionals who can adapt micronutrients to reduce the risks of nutritional deficiencies, especially in relation to vitamin $B 12$ and iron. CONCLUSION: It is possible to include the vegetarian diet in the eating habits of children, as long as the accompaniment of a qualified health professional, able to adapt all the essential nutrients for this age.

Keywords: Benefts. Complementary Feeding. Vegetarian Diet. 


\section{INTRODUÇÃO}

O período em que se inicia a introdução da Alimentação Complementar é considerado de rápido crescimento e desenvolvimento infantil concomitante a novas e grandes mudanças dietéticas, com exposição a novos alimentos, sabores e experiências alimentares. A Alimentação Complementar deve incluir os grupos alimentares para uma nutrição completa e balanceada como: grãos, raízes e tubérculos, leguminosas e oleaginosas, produtos lácteos, carnes e ovos, frutas e legumes. (NEVES et al., 2020). Contudo, a alimentação de uma criança nas primeiras fases de sua vida tem repercussões a curto e longo prazo. É sabido, por exemplo, que um adequado hábito alimentar durante os primeiros anos da infância pode prevenir desnutrição, deficiência de certos micronutrientes e também sobrepeso e obesidade, sendo o déficit causado por hábitos alimentares inadequados é dificilmente revertido após os dois anos de idade da criança. (GURMINI et al., 2017).

O consumo de uma dieta vegetariana pode resultar em menor ingestão de nutrientes importantes para o desenvolvimento cognitivo. $\mathrm{O}$ consumo de uma dieta vegetariana durante a gravidez já foi associado a menores concentrações de cobalamina e alguns ácidos graxos poliinsaturados que são necessários para o desenvolvimento e função normais do cérebro. No entanto, estudos mostram que a adoção de uma dieta vegetariana durante a gravidez não apresentou efeitos negativos na função cognitiva dascrianças de até 6 anos de idade (COFNAS, 2018; BURDGE, 2017).

O crescimento dessas crianças pode ser acompanhados por instrumentos como as curvas de crescimento preconizadas pela OMS (Organização Mundial de Saúde), onde será analisado o estado nutricional do mesmo pelas medidas antropométricas comopeso, altura/comprimento, IMC e perímetro cefálico (BOMFIM, C.; MORGADO, F.; BUENO, A. 2018). Essas medidas antropométricas não apresentam divergências em crianças onívoras e vegetarianas, pois desde que 
sejam feitas com responsabilidade e cuidado em manter uma dieta balanceada, não há incompatibilidade entre a ingestão de macronutrientes e micronutrientes nesses dois grupos (WEDER S. et al. 2019).

A dieta vegetariana pode levar a um consumo menor de alguns micronutrientes como ferro, cálcio, zinco e vitamina B12 que são melhor biodisponíveis nos alimentos de origem animal (SBP, 2017). A oferta correta dos nutrientes por um profissional capacitado é muito importante para que a criança não sofra com as carências nutricionais, que podem ser evitadas com acompanhamento e planejamento de macronutrientes e micronutrientes, diversificação e qualidade nas refeições, assegurando assim o desenvolvimento de forma saudável (PIMENTEL D. et al. 2018).

O padrão alimentar vegetariano se bem planejado apresenta benefícios à saúde, como a prevenção contra certas doenças crônicas, sendo elas a obesidade, doenças cardiovasculares e câncer. (MARTINS, S. 2019).

É viável a dieta vegetariana infantil, desde que adequada aos padrões necessários de desenvolvimento de cada fase da criança, podendo ser iniciado desde o início da infância, até a chegada da vida adulta. Para isso, é necessária a avaliação do plano alimentar vegetariano, pelo profissional nutricionista, cujo papel é considerar cadavariável, elaborando uma estratégia individualizada para cada etapa do ciclo da vida. O posicionamento da American Dietetic Association (CRAIG, MANGELS, 2009) é de que dietas vegetarianas bem planejadas são saudáveis e nutricionalmente adequadas, podendo haver benefícios à saúde e tratando enfermidades. O estudo teve como objetivo reunir evidências científicas acerca dos benefícios e malefícios do vegetarianismo na introdução alimentar de crianças até a idade pré-escolar para fornecer informações aos profissionais de saúde para que possam auxiliar no processo de transmissão de conhecimentos para cuidadores que desejam incorporar a dieta vegetariana aos hábitos alimentares das crianças nesse período tão importante que é a introdução alimentar. 


\section{METODOLOGIA}

A pesquisa trata-se de um estudo de revisão integrativa da literatura, de naturezaqualitativa e exploratória. A busca dos artigos foi realizada em quatro bases de dados, a Literatura Latino Americana e do Caribe em Ciências da Saúde (Lilacs), Scientific Electronic Library Online (SciELO), National Library of Medicine (PubMED) e o Google Acadêmico.

Para o levantamento de dados foram utilizados os seguintes descritores: "dieta vegetariana", "vegetarianismo", "desenvolvimento cognitivo", "desenvolvimento e crescimento", "crianças", "introdução alimentar", "infância", "deficiências nutricionais", "riscos e benefícios" no idioma português cadastrados nos Descritores em Ciências da Saúde (DeCS) e inglês, de acordo com o Medical Subject Headings (MeSH) "food complementary","vegetarianism", "cognitive development", "development and growth", "deficiency diseases", "children”, "diet vegetarian", "risks and benefits" adaptados de acordo com a base de dados utilizada.

Seguiu-se no desenvolvimento do estudo cinco etapas: a primeira busca foi iniciada pelos descritores: "alimentação complementar vegetariana"; a segunda com os descritores "desenvolvimento cognitivo", "dieta vegetariana" e "crianças" conectados pelo operador booleano AND; a terceira com os descritores "dieta vegetariana", "crianças", "desenvolvimento", "deficiência nutricional” e "crescimento"; a quarta com os descritores "pré escolares", "vegetarianismo", "crianças", "dieta vegetariana" e "deficiência nutricional" conectados pelo booleano AND; e a quinta quinta com os descritores "dieta vegetariana", "infância", "crianças", "riscos e benefícios".

A busca foi realizada no mês de fevereiro de 2021, na qual foram selecionados69 artigos. Após a aplicação dos critérios de inclusão e exclusão foram eleitos para esta 
Figura 1. Fluxograma das etapas de verificação na busca e seleção dos estudos incluídosna revisão. Brasil, 2021.

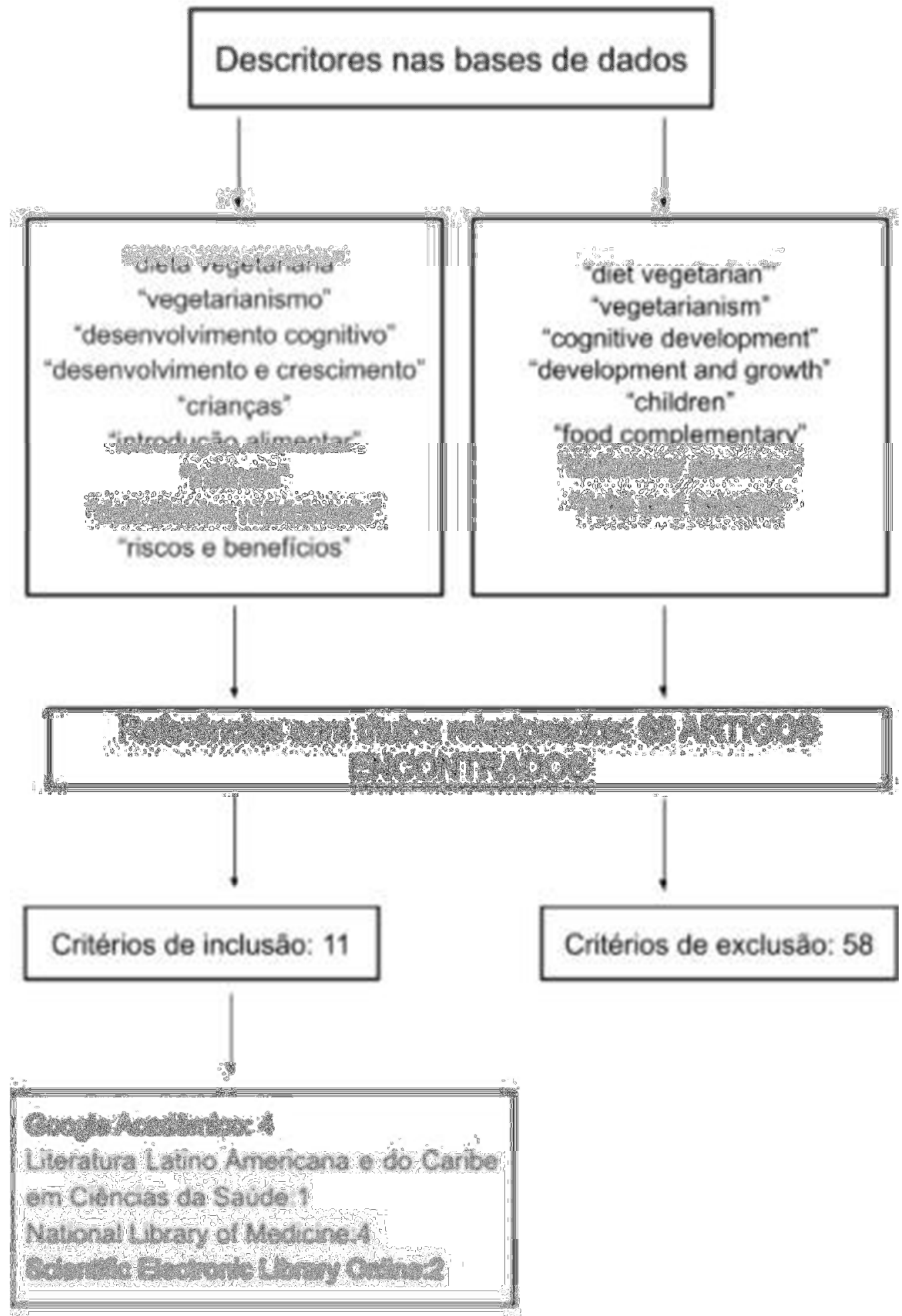

Fonte: Dados da pesquisa, 2021. 
Os critérios de inclusão utilizados para a seleção apresentada na figura acima foram: artigos originais, indexados nas bases de dados selecionadas, nos idiomas: português e inglês; publicados entre os anos de 2011 e 2021. A escolha desse período atendeu ao critério de temporalidade, em que se considerou o recorte de dez anos, por se tratar de publicações mais atuais relativas aos temas. Foram excluídas publicações de teses, anais de congressos e simpósios, dissertações, monografias e artigos que não estavam relacionados ao tema. Na primeira avaliação foi efetuada a leitura dos resumos dos textos, para verificação da adequação ao tema e duplicidade, seguida de leitura exaustiva e classificação daqueles que constituíram as categorias de análise.

\section{RESULTADOS}

Apresenta-se as produções científicas que englobam os temas de desenvolvimento e crescimento físico em crianças vegetarianas, no período de 2011 a 2021 segundo autor, ano, título, objetivo, tipo de estudo e resultados. 
Tabela de artigos encontrados com o referido tema: Introdução Alimentar Vegetariana.

\begin{tabular}{|c|c|c|c|c|}
\hline Autor/Ano & Título & Objetivo & $\begin{array}{l}\text { de } \\
\text { do }\end{array}$ & Resultado \\
\hline $\begin{array}{l}\text { PIM! } \\
\text { et al }\end{array}$ & $\begin{array}{l}\text { Alimentação } \\
\text { Vegetariana } \\
\text { nos primeiros } \\
\text { anos de vida: } \\
\text { Consideraçõe } \\
\text { s } \\
\text { Orientações/ } \\
\text { Vegetarian } \\
\text { diets in first } \\
\text { years } \\
\text { life:considerati } \\
\text { ons and } \\
\text { orientations. }\end{array}$ & \begin{tabular}{|lr} 
Apresentar & um \\
compilado & de \\
informações & \\
válidas & e \\
cientificamente & \\
fundamentadas que \\
permitam orientar a \\
diversificação & \\
alimentar & do \\
lactente & no \\
contexto de r uma \\
alimentação \\
vegetariana.
\end{tabular} & $\begin{array}{l}\text { Artigo } \\
\text { revisão }\end{array}$ & 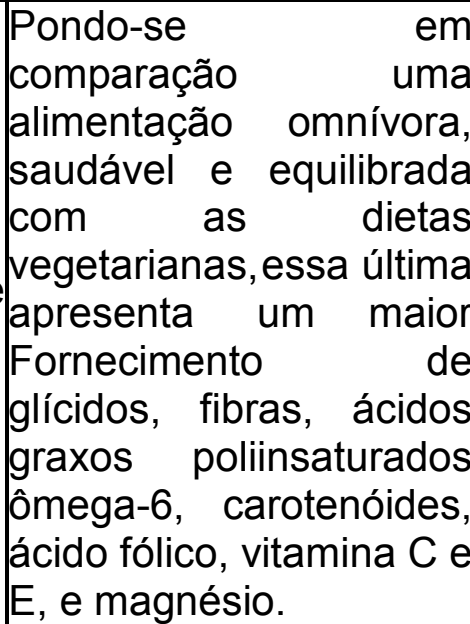 \\
\hline $\begin{array}{l}\text { ALI } \\
2014\end{array}$ & $\begin{array}{l}\text { Introdução } \\
\text { alimentar em } \\
\text { crianças } \\
\text { vegetarianas } \\
\text { Revisão de } \\
\text { literatura/ } \\
\text { Food release } \\
\text { for vegetarian } \\
\text { children }\end{array}$ & 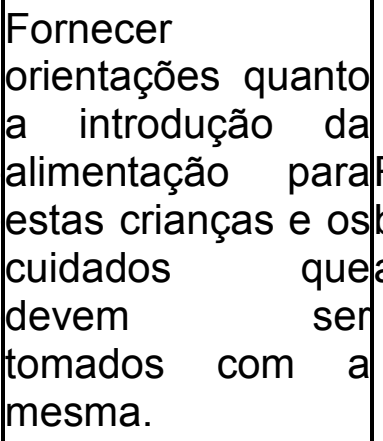 & & $\begin{array}{l}\text { Teve-se que a } \\
\text { suplementação de uma } \\
\text { criança vegetariana é } \\
\text { seme-lhante à de uma } \\
\text { não vegetariana, salvo } \\
\text { pequenas exceções, } \\
\text { como a vitamina B12. }\end{array}$ \\
\hline $\begin{array}{l}\text { BETTINELL } \\
\text { E. et a } \\
2019 .\end{array}$ & $\begin{array}{l}\text { Knowledge of } \\
\text { Health } \\
\text { Professionals } \\
\text { Regarding } \\
\text { Vegetarian } \\
\text { Diets from } \\
\text { Pregnancy to } \\
\text { Adolescence: } \\
\text { An } \\
\text { Observational } \\
\text { Study }\end{array}$ & $\mid \begin{array}{lr}\text { Investigar } & \\
\text { conhecimento dos } \\
\text { profissionais } & \text { de } \\
\text { saúde sobre } \\
\text { adoção de dietas } \\
\text { vegetarianas } \\
\text { gravidez da } \\
\text { adolescência. }\end{array}$ & $\begin{array}{l}\text { Estudo } \\
\text { observacio } \\
\text { nal/ } \\
\text { transversal }\end{array}$ & $\begin{array}{l}\text { Os resultados do estudo } \\
\text { indicam que os } \\
\text { profissionais de saúde } \\
\text { fazem não possuem } \\
\text { conhecimento completo } \\
\text { e exaustivo sobre dietas } \\
\text { vegetarianas e faltam } \\
\text { informações sobre } \\
\text { saúde, resultados e a } \\
\text { adoção de uma dieta } \\
\text { vegetariana ao longo } \\
\text { dos diferentes ciclos de } \\
\text { vida e nutrientes. }\end{array}$ \\
\hline
\end{tabular}

Tabela de artigos encontrados com o referido tema: benefícios e deficiências de macronutrientes e micronutrientes em crianças no uso da alimentação vegetariana. 


\begin{tabular}{|c|c|c|c|c|}
\hline Autor/Ano & Título & Objetivo & $\begin{array}{l}\text { Tipo de } \\
\text { Estudo }\end{array}$ & Resultado \\
\hline $\begin{array}{l}\text { SOCIEDAD E } \\
\text { BRASILEIR A } \\
\text { DE } \\
\text { PEDIATRIA, } \\
2017 .\end{array}$ & $\begin{array}{lr}\text { Vegetarianis } \\
\text { mo } \\
\text { infância na } \\
\text { adolescência }\end{array}$ & $\mid \begin{array}{l}\text { Orientar } \\
\text { quanto ao } \\
\text { vegetarianism } \\
\text { o, seus riscos } \\
\text { e benefícios } \\
\text { na infância e } \\
\text { adolescência. }\end{array}$ & $\begin{array}{l}\text { Artigo } \\
\text { Revisão }\end{array}$ & \begin{tabular}{|lr} 
A orientação quanto a \\
introdução alimentar é a \\
mesma para lactentes não \\
vegetarianos. A orientação \\
profissional quanto à oferta \\
de calorias adequadas, \\
suplementação & de \\
micronutrientes e vitaminas \\
(quando indicado) e o \\
acompanhamento & é \\
importante para \\
desenvolvimento infantil \\
adequado.
\end{tabular} \\
\hline $\begin{array}{l}\text { BARRANH A, } \\
\text { S. G. W. } 2017\end{array}$ & $\begin{array}{l}\text { Alimentação } \\
\text { vegetariana } \\
\text { em idade } \\
\text { pediátrica: } \\
\text { riscos, } \\
\text { benefícios e } \\
\text { recomendaç } \\
\text { ões. }\end{array}$ & 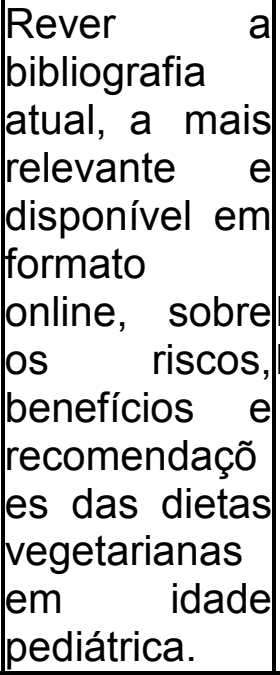 & Revi & $\begin{array}{l}\text { A dieta vegetariana e todas } \\
\text { as suas variantes, podem } \\
\text { seradotadas em Pediatria se } \\
\text { planejadas, e caso } \\
\text { necessário suplementadas, } \\
\text { não causando qualquer } \\
\text { dano e até podendo trazer } \\
\text { benefícios a longo prazo. }\end{array}$ \\
\hline $\begin{array}{l}\text { INTERNATI } \\
\text { ONAL } \\
\text { JOURNAL OF } \\
\text { NUTROLO } \\
\text { GY } 2018\end{array}$ & $\begin{array}{l}\text { Riscos e } \\
\text { benefícios } \\
\text { da dieta } \\
\text { vegetariana } \\
\text { em crianças }\end{array}$ & $\mid \begin{array}{lr}0 & \text { trabalho } \\
\text { visa avaliar } & \text { os } \\
\text { benefícios } & \text { e } \\
\text { riscos } & \text { da } \\
\text { dieta } & \\
\text { vegetariana } \\
\text { durante } \\
\text { infância. }\end{array}$ & $\begin{array}{l}\text { Artigo } \\
\text { revisão }\end{array}$ & \begin{tabular}{|lrr|} 
Entre & estes & \multicolumn{2}{r|}{ trabalhos, } \\
poucos & malefícios & foram \\
retratados. Esse r grupo \\
normalmente possui um \\
menor aporte energético \\
durante seus primeiros anos \\
de vida, no entanto esta \\
diferença tende a igualar-se \\
às ranças r não \\
vegetarianas até os 10 anos \\
de idade.
\end{tabular} \\
\hline
\end{tabular}




\begin{tabular}{|c|c|c|c|c|}
\hline $\begin{array}{l}\text { MARTINS, D } \\
\text { FARIA, } \\
\text { LOUREIRO, } \\
\text { H. } 2019\end{array}$ & $\left\{\begin{array}{l}\text { Alimentação } \\
\text { vegetariana } \\
\text { na criança e } \\
\text { no } \\
\text { adolescente }\end{array}\right.$ & $\begin{array}{l}\text { O objetivo } \\
\text { deste artigo é } \\
\text { identificar os } \\
\text { motivos que } \\
\text { provocam } \\
\text { uma adesão } \\
\text { ao padrão } \\
\text { alimentar } \\
\text { vegetariano e } \\
\text { identificar os } \\
\text { efeitos sobre } \\
\text { a saúde deste } \\
\text { padrão } \\
\text { alimentar nas } \\
\text { crianças } \\
\text { adolescentes. }\end{array}$ & Artigo & $\begin{array}{l}\text { o padrão alimentar em } \\
\text { questão apresenta alguns } \\
\text { benefícios para a saúde, } \\
\text { nomeadamente r na } \\
\text { prevenção de doenças como } \\
\text { de obesidade, doenças } \\
\text { cardiovasculares, diabetes } \\
\text { tipo } 2 \text { e cancro. Por fim, o } \\
\text { padrão alimentar } \\
\text { vegetariano, se for } \\
\text { corretamente planeado, } \\
\text { pode fornecer as } \\
\text { necessidades nutricionais de } \\
\text { crianças e adolescentes. }\end{array}$ \\
\hline
\end{tabular}

Tabela de artigos encontrados com o referido tema: desenvolvimento cognitivo, crescimento físico e dieta vegetariana em crianças.

\begin{tabular}{|c|c|c|c|c|}
\hline Autor/Ano & Título & Objetivo & $\begin{array}{l}\text { Tipo de } \\
\text { Estudo }\end{array}$ & Resultado \\
\hline $\begin{array}{l}\text { PEPPER, } \\
\text { M., } \quad \text { BLACK, } \\
\text { M., } 2011 \\
\text { SHENG } \quad \text { ET } \\
\text { AL., } 2019\end{array}$ & $\begin{array}{l}\text { B12 in fetal } \\
\text { development } \\
\text { Effects of } \\
\text { dietary } \\
\text { intervention } \\
\text { on vitamin } \\
\text { B12 status } \\
\text { and cognitive } \\
\text { level of 18- } \\
\text { month old } \\
\text { toddlers in } \\
\text { high-poverty } \\
\text { areas: a } \\
\text { cluster- } \\
\text { randomized } \\
\text { controlled trial }\end{array}$ & \begin{tabular}{|l} 
Revisar \\
mecanismos da \\
influência \\
cobalamina da \\
desenvolvimento \\
infantil Observar \\
os efeitos de \\
diferentes \\
alimentos \\
complementares \\
nos níveis de \\
vitamina B12 e \\
nível cognitivo de \\
crianças de áreas \\
de baixa renda na \\
China
\end{tabular} & $\begin{array}{l}\text { Revisão } \\
\text { Ensaio } \\
\text { clínico } \\
\text { randomizad } \\
\text { o }\end{array}$ & $\begin{array}{lrr}\text { A deficiência } & \text { de } & \text { B12 } \\
\text { durante a } & \text { infância, } \\
\text { comum em } & \text { crianças } \\
\text { vegetarianas, } & & \text { Pode } \\
\text { comprometer } & & \text { o } \\
\text { desenvolvimento cognitivo } \\
\text { e motor Cereais } & \text { de arroz } \\
\text { fortificados } & & \text { com } \\
\text { micronutrientes } & \text { podem } \\
\text { ajudar a melhorar os } \\
\text { níveis de vitamina B12 } \\
\text { em crianças que adotam } \\
\text { dietas vegetarianas e seu } \\
\text { nível cognitivo. }\end{array}$ \\
\hline $\begin{array}{l}\text { BOMFIM, } \\
\text { C.; } \\
\text { MORGADO, } \\
\text { F.; BUENO, } \\
\text { A. } 2018\end{array}$ & $\begin{array}{l}\text { Uma revisão } \\
\text { bibliográfica: } \\
\text { os } \\
\text { da impactos } \\
\text { vegana r em } \\
\text { crianças. }\end{array}$ & $\begin{array}{l}\text { Estudar os riscc } \\
\text { da dieta vegar } \\
\text { em crianças. }\end{array}$ & $\begin{array}{l}\text { Revisão } \\
\text { bibliográf } \\
\text { ica }\end{array}$ & $\begin{array}{l}\text { A dieta vegana sem } \\
\text { suplementaçã o não é } \\
\text { capaz de nutrir uma } \\
\text { criança em desenvolvime } \\
\text { nto. }\end{array}$ \\
\hline
\end{tabular}




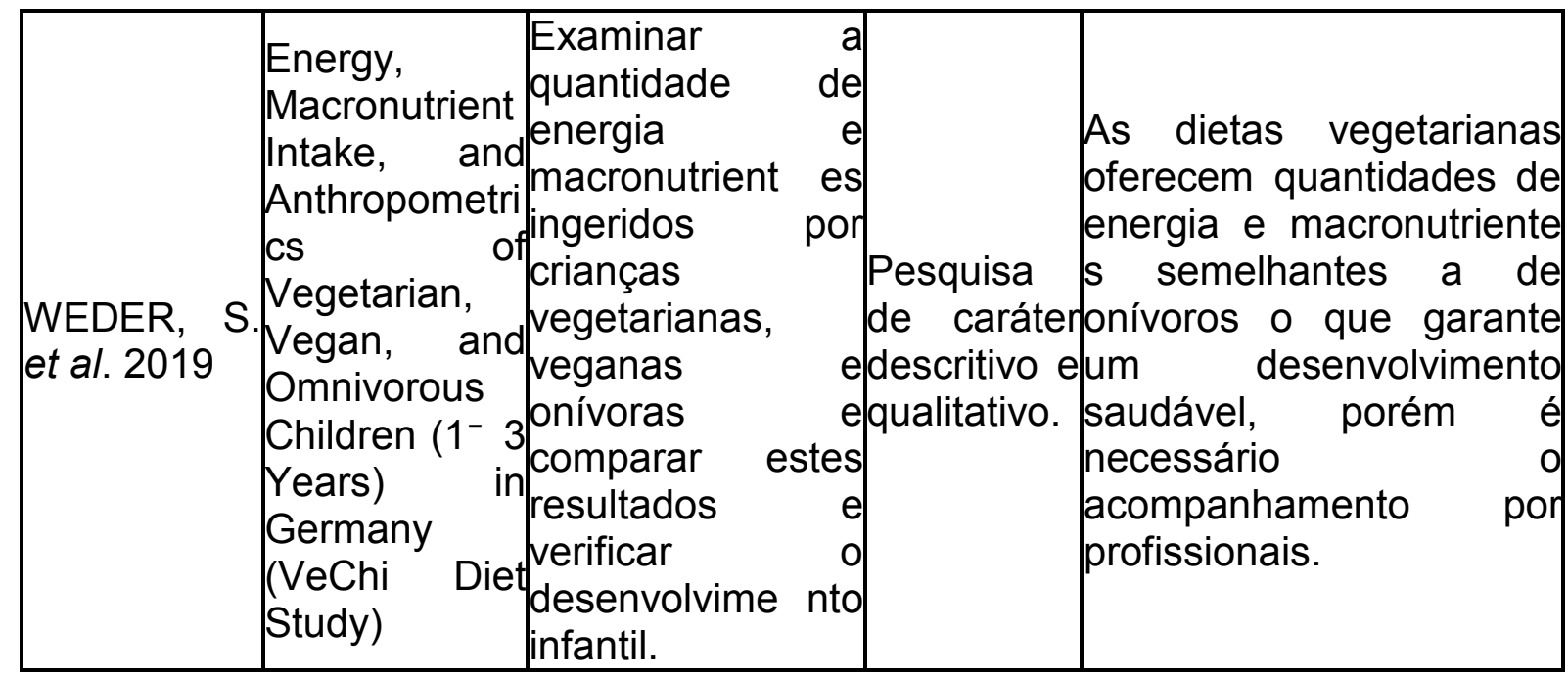

\section{DISCUSSÃO}

Segundo Pimentel et al., (2018) pondo-se em comparação uma alimentação omnívora, saudável e equilibrada com as dietas vegetarianas, essa última apresenta um maior fornecimento de glícidos, fibras, ácidos graxos poliinsaturados ômega6, carotenóides, ácido fólico, vitamina $\mathrm{C}$ e E, e magnésio. Assim sendo para o grupo dos lactentes na fase da introdução alimentar não é diferente, A European Society for Paediatric Gastroenterology Hepatology and Nutrition (ESPGHAN), a American Dietetic Association (ADA), a Academy of Nutrition and Dietetics (AND) e a American Academy of Pediatrics (AAP) afirmam que a implementação de um regime alimentar vegetariano bem planejado, mesmo na diversificação/introdução alimentar, é sim passível de satisfazer as necessidades nutricionais dos lactentes e permitir o seu normal desenvolvimento estato-ponderal e cognitivo. A vista disso, foi observado através do estudo de Ali et.al., (2014) que esses benefícios quanto a presença de compostos favoráveis para o organismo dos praticantes da dieta vegetariana relatados nos artigos anteriores trazem consigo também efeitos clínicos positivos, como uma menor incidência de casos de hipertensão e doenças coronarianas, obesidade, alguns tipos de câncer e ainda o diabetes com início na fase adulta.

Todavia Bettinelli et al., (2019) trás em seu estudo sobre o risco potencial que é as pessoas que vem a adotar esse tipo de dieta somente porque está na moda ou 
devido aquestões éticas relacionadas ao bem-estar animal, sem ser adequadamente educados e informados sobre os efeitos potencialmente negativos caso essas dietas não foremadequadamente planejadas e principalmente nesse público da IA.

Entretanto, são utilizados diversos instrumentos para avaliar o crescimento de uma criança e como um desses a OMS (Organização Mundial da Saúde), a MS (Ministério da Saúde) e a SBP (Sociedade Brasileira de Pediatria) definem curvas de crescimento infantil para analisar o estado nutricional e de desenvolvimento físico, por meio de instrumentos como medidas antropométricas e análises de evolução. De acordo com BOMFIM, C.; MORGADO, F. BUENO, A. (2018) uma dieta vegetariana restrita não é capaz de fornecer o necessário, para um bom desenvolvimento físico, portanto a criança teria que ser suplementada ou ter a ingestão aumentada para conseguir atingir o desenvolvimento pleno. No estudo ocorrido na Alemanha WERDER S. et al (2019) não foram encontrados dados antropométricos diferentes entre crianças vegetarianas e veganas em comparação com as crianças onívoras, o que nos leva a conclusão de que ambas teriam um crescimento normal.

O melhor desempenho cognitivo parece estar associado ao consumo adequado de proteínas e micronutrientes, mais especificamente zinco e ferro, principalmente em dietas onívoras, pois esses micronutrientes se apresentam na forma mais biodisponível nos alimentos de origem animal. Os principais estudos sugerem que mesmo com planejamento alimentar e suplementação, veganos tendem a apresentar níveis limítrofes ou deficientes de vitamina B12 (cobalamina) bem como EPA (ácido eicosapentaenoico) e DHA (ácido docosahexaenoico) componentes do ômega 3 , o que pode acarretar prejuízos no desenvolvimento cognitivo de crianças, com queda do desempenho escolar, fraqueza, cansaço, depressão, transtornos de déficit de atenção e hiperatividade. O DHA é um nutriente essencial para o desenvolvimento do cérebro e da retina, especialmente nos primeiros 2 anos de vida (BURDGE, 2017).

As dietas vegetarianas são consideradas saudáveis, desde que sejam planejadas por um profissional competente, e seja adequada para cada criança, diminuindo assim os riscos e trazendo equilíbrio na oferta de nutrientes. A carência de nutrientes específicos, como vitaminas e minerais pode prejudicar o crescimento 
e desenvolvimento infantil, além de trazer graves consequências que podem perdurar por toda a vida. Não é possível afirmar que todos os macronutrientes e micronutrientes consumidos serão suficientes para atender a exigência precisa na nutrição da criança, portanto caso se torne necessário para atingir o consumo dietético infantil é indispensável a suplementação durante a infância (BARROSO, 2017).

A criança menor de 2 anos a 6 anos vegetariana necessita de $10 \%$ a $20 \%$ a mais de proteína dependendo da idade, o ferro é suplementado a partir dos 4 meses em todas as crianças para que atinja níveis consideráveis, quando existem dúvidas acerca do déficit de vitaminas e minerais a suplementação é realizada. A diversificação alimentar é a melhor opção para a criança, oferecendo as melhores fontes alimentares de origem vegetal (SBP, 2017).

As evidências científicas relacionadas ao padrão alimentar vegetariano vem aumentando ao longo dos anos, principalmente relacionado à prevenção de doenças crônicas que afetam a sociedade em todas as suas esferas, mostrando que uma dieta vegetariana bem planejada e adequada, ainda na infância pode reduzir a incidência de algumas doenças na idade adulta, como obesidade, que é um problema de saúde pública que está aumentando em áreas urbanizadas de países em desenvolvimento, o estado de inflamação, doenças cardiovasculares, diabetes tipo 2, câncer, osteoporose e menor exposição das crianças a antibióticos veterinários. (MARTINS, D. et al. 2019).

Segundo o International Journal of Nutrology (2018) esse grupo normalmente possui um menor aporte energético durante seus primeiros anos de vida, no entanto esta diferença tende a igualar-se às crianças não vegetarianas até os 10 anos de idade. Além disso, existe a possibilidade de que este grupo desenvolva deficiência de vitamina B12, assim como de ácidos graxos,ômega 3, gorduras saturadas, ferro, zinco e vitamina D. Porém, estas deficiências são facilmente reestabelecidas com suplementação. Em contrapartida, pelo fato de que essas crianças tendem a ingerir mais frutas, verduras, cereais, legumes e sementes, seus níveis de vitamina $A, C, E$, tiamina, ácido fólico, riboflavina, fibras, magnésio, cobre e ácidos graxos do tipo ômega 6 costumam ser maiores. Além disso, foi comprovado que a manutenção da dieta vegetariana evidencia uma menor incidência de câncer de estômago, ovário, 
bexiga, tecido linfático e hematopoiético do que em indivíduos onívoros. Dentre os fatores fisiológicos, os níveis séricos de diversos tipos de antioxidantes são maiores, o que leva a maior proteção contra a oxidação das lipoproteínas e maior estabilidade genômica.

\section{CONCLUSÃO}

É possível incluir a dieta vegetariana no hábito alimentar de crianças desde que seja realizado o acompanhamento de um profissional de saúde habilitado que possa adequar todos os macro e micronutrientes que são fundamentais desde a introdução alimentar até a idade pré-escolar. Para que o desenvolvimento físico e cognitivo da criança se desenvolva normalmente são necessários vários fatores, um deles a alimentação adequada para cada período da infância, onde temos picos de alta demanda de alguns nutrientes como são os casos da vitamina B12 e do ferro.

\section{REFERÊNCIAS BIBLIOGRÁFICAS}

ALI G. et al.Introdução Alimentar em crianças vegetarianas-Revisão de Literatura. UNINGÁ Review, v. 20, n. 3, 2014. Acesso em: 23 fev 2021.

BARRANHA, S.G.W. Alimentação vegetariana em idade pediátrica: riscos, benefícios e recomendações. Instituto de Ciências Biomédicas Abel Salazar. Universidade do Porto. 2017. Disponível em: https://repositorio- aberto.up.pt/bitstream/10216/109780/2/238265.pdf. Acesso em: 23 fev. 2021.

BETTINELLI, M.E.; et al., Knowledge of Health Professionals Regarding Vegetarian Diets from Pregnancy to Adolescence: An Observational Study. Nutrients 2019, 11(5): 1149. Disponível em: https://doi.org/10.3390/nu11051149. Acesso em: 20 de fev. 2021.

BURDGE, G.C.; TAN, S.Y.; HENRY, C. Long-chain n-3 PUFA in vegetarian women: a metabolic perspective. J Nutr Sci. vol.6, ed.6, 2017. Disponível em: https://www.ncbi.nlm.nih.gov/pmc/articles/PMC5705809/pdf/S2048679017000623a.pdf. Acesso em 18 fev. 2021.

BOMFIM, C.; MORGADO, F.; BUENO, A. Uma revisão bibliográfica: os impactos da dieta vegana em crianças. Revista da Faculdade de Medicina de Teresópolis, Teresópolis, v. 2, n. $1, \quad$ p. $1-121, \quad 2018 . \quad 2$ Disponível $\quad$ em: http://www.revista.unifeso.edu.br/index.php/faculdadedemedicinadeteresopolis/article/v iew/613/416. Acesso em: 12 de fev. 2021. 
COFNAS N. Is vegetarianism healthy for children?. Crit Rev Food Sci Nutr. 2019. Disponível em: https://pubmed.ncbi.nlm.nih.gov/29405739/. Acesso em: 23 fev.2021.

GUEDES A.et al. INTRODUÇÃO ALIMENTAR EM CRIANÇAS VEGETARIANAS - REVISÃO DE LITERATURA. UNINGÁ Review, v. 20, n. 3, 2014. Disponível em: http://www.scielo.mec.pt/scielo.php?script=sci_arttext\&pid=S2183-

59852019000300009\&Ing=pt\&nrm=iso. ISSN 2183-5985. Acesso em: 20 de fev.2021.

GURMINI, J. et al. Análise da Alimentação complementar em crianças entre 0 e 2 anos de escolas públicas. Rev. Med. UFPR 4(2): 55-60 Abr-jun/2017. Acesso em: 23 fev 2021.

MARTINS, D.; S. FARIA, A. ; LOUREIRO.H. Alimentação vegetariana na criança e no adolescente. Acta Port Nutr, Porto, n. 18, p. 50-53 jul. 2019. Disponível em $<$ http://www.scielo.mec.pt/scielo.php?script=sci_arttext\&pid=S2183-

$59852019000300009 \&$ lng=pt\&nrm=iso>. Acesso em 08 fev. 2021.

NEVES, Renata Oliveira et al . A paridade pode influenciar na alimentação do lactente nos primeiros seis meses de vida?. Ciênc. saúde coletiva, Rio de Janeiro, v. 25, n. 11,

p. 4593-4600, Nov. 2020. Disponivel em: $<$ http://www.scielo.br/scielo.php?script=sci_arttext\&pid=S1413-

81232020001104593\&lng=en\&nrm=iso>. Acesso em: 25 Fev. 2021.

NUNES, Bruna dos Santos; GUBERT, Muriel Bauermann; BORTOLINI, Gisele Ane. AS RECOMENDAÇÕES OFICIAIS SOBRE AMAMENTAÇÃO E ALIMENTAÇÃO COMPLEMENTAR SÃO ACESSIIVEIS E CONHECIDAS PELOS PROFISSIONAIS DE SAÚDE BRASILEIROS? DEMETRA: Alimentação, Nutrição \& Saúde, [S.I.], v.14, p. E43327,nov.2019. ISSN:2238-913X. Disponível em: <https://www.epublicacoes.uerj.br/index.php/demetra/article/view/43327>. Acesso em: 25 fev. 2021. doi:https://doi.org/10.12957/demetra.2019.43327. Acesso em: 15 fev 2021.

PEPPER, M.R.; BLACK, M.M. B12 in fetal development. Semin Cell Dev Biol. vol.22, ed.6, 2011. Disponível em: PIMENTEL., D. et al.; Alimentação vegetariana nos primeiros anos de vida: considerações e orientações. Acta Portuguesa de Nutrição, n. 14, p. 10-17, 2018. Disponível em: <https://actaportuguesadenutricao.pt/wpcontent/uploads/2018/11/03_Alimenta\%C3\%A7\%C3\%A3o-vegetariana-nos-primeiros- anos-devida-considera\%C3\%A7\%C3\%B5es-e-orienta\%C3\%A7\%C3\%B5es.pdf>. Acesso em: 22 de fev. 2021.

REITER,M.G.R. et al. Riscos e Benefícios da Dieta Vegetariana em Crianças. International Journal of Nutrology 2018; 11(S 01): S24-S327; DOI: 10.1055/s-0038- 1675010. Disponível em: https://www.thieme- connect.com/products/ejournals/abstract/10.1055/s-0038-1675010. Acesso em: 23 de fev. 2021.

SOCIEDADE BRASILEIRA DE PEDIATRIA. Vegetarianismo na infância e adolescência. Guia Prático de Atualização. Sociedade Brasileira de Pediatria. 2017, $\mathrm{n}^{\circ}$ 4. Disponível em: https://www.sbp.com.br/fileadmin/user_upload/Nutrologia_-_Vegetarianismo_Inf_e_Adolesc.pdf. Acesso em: 24 fev. 2021.

SHENG, X; WANG, J.L.F.; OUYANG, F.M.J. Effects of dietary intervention on vitamin $B_{12}$ status and cognitive level of 18-month-old toddlers in high-poverty areas: a cluster-randomized controlled trial. BMC Pediatr. vol.19, 2019. Disponível em: https://www.ncbi.nlm.nih.gov/pmc/articles/PMC6743151/pdf/12887_2019_Article_171 6.pdf. Acesso em 18 fev. 2021.

SILVA, M. D. C.; TOMADA, I. ; R., C.. Alimentação Vegetariana nos Primeiros Dias de vida: considerações e orientações. 2017. 27 f. Faculdade de Ciências da Nutrição e Alimentação do Porto, Porto, 2017. Disponivel em: <https://repositorio- 
aberto.up.pt/bitstream/10216/107062/2/210284.pdf> . Acesso em: 13 fev 2021.

Schürmann S, Kersting M, Alexy U. Vegetarian diets in children: a systematic review. Eur J Nutr., Agosto, Epub 2017. Disponível em: <https://pubmed.ncbi.nlm.nih.gov/28299420/>. Acesso em: 23 fev.2021.

WEDER,W. Et al. Energy, Macronutrient Intake, and Anthropometrics of Vegetarian, Vegan, and Omnivorous Children (1- 3 Years) in Germany (VeChi Diet Study). Nutrients. 2019;11(4):832. Published 2019 Apr 12. Disponível em: https://pubmed.ncbi.nlm.nih.gov/31013738/. Acesso em: 10 de fev. 2021. 